\title{
Worst-Case Deterministic Delay Bounds for Arbitrary Weighted Generalized Processor Sharing Schedulers
}

\author{
Robert Szabó, Peter Barta, Felician Németh, and jozsef Bíró
}

High Speed Networks Laboratory

Dept. of Telecommunications and Telematics, Technical University of Budapest

Stoczek u. 2, H-1111, Budapest, Hungary

\{szabor, barta, nemethf, biro\}@ttt-atm.ttt.bme.hu

\begin{abstract}
Generalized Processor Sharing (GPS) is realized as the most important ideal fluid scheduling discipline in guaranteed QoS networks. The well-known problem of GPS-based rate-proportional servers is the bandwidth delay coupling which leads to inefficient resource utilization. In this paper we introduce the concept of non rate-proportional (or arbitrary) weighting of sessions in GPS systems. Such an approach in a GPS node of network constrained by leaky buckets can handle bandwidth and delay parameters independently, thus allowing better utilization of network resources. Moreover, we show that even under the traditional bandwidth delay coupled system (rate-proportional weighting) it is possible to determine tighter delay bounds of sessions than that of presented in earlier papers. A numerically inexpensive algorithm, which works in any arbitrary weighted GPS system is also presented for computing delay bounds. Besides the analytical work numerical examples are also shown.
\end{abstract}

\section{Introduction}

The primary QoS requirements of applications in multi-service telecommunication networks such as end-to-end packet delay, loss and bandwidth must be provided on a per-connection basis for guaranteed performance services. A network meets these requirements primarily by appropriately scheduling its resources, which in turn assumes the use of proper traffic scheduling algorithms within individual network elements (switches, routers) [1,2].

Traffic scheduling algorithms usually operate on packet/cell level and assume there are different sessions, which traffic is to be scheduled for. The task of service disciplines at switching nodes is to control the order in which incoming packets are served in order to provide (end-to-end) performance bounds. Based on delay and fairness properties, one of the most significant scheduling algorithms is Generalized Processor Sharing (GPS) [5]6], which is a generalized version of Uniform Processor Sharing [4. The packet-by-packet version of GPS (PGPS) is essentially the same as Weighted Fair Queuing (WFQ) [3], however they were independently developed. 
A GPS server serving $N$ sessions is characterized by $N$ positive real numbers, $\phi_{1}, \phi_{2}, \ldots, \phi_{N}$. The server operates at a fixed rate $r$ and is work-conserving, that is, never idle whenever there are data to send. Let $W_{i}\left(t_{1}, t_{2}\right)$ be the amount of session $i$ traffic served in the interval $\left[t_{1}, t_{2}\right]$, then a GPS server is defined as one for which

$$
\frac{W_{i}\left(t_{1}, t_{2}\right)}{W_{j}\left(t_{1}, t_{2}\right)} \geq \frac{\phi_{i}}{\phi_{j}}, \quad j=1,2, \ldots, N
$$

for any session $i$ that is continuously backlogged 1 in the interval $\left[t_{1}, t_{2}\right]$. The immediate consequence of this definition is that every session has a minimum guaranteed service rate, that is $g_{i}=\frac{\phi_{i}}{\sum_{j=1}^{N} \phi_{j}} r$ [5].

Under GPS scheduling each session has a separate queue served by the corresponding guaranteed rate in FIFO manner. The input traffic of every session can be shaped by a leaky bucket that is characterized by a token pool depth $(\sigma)$ and a token generation rate $(\rho)$. The amount of incoming traffic in the interval $\left[t_{1}, t_{2}\right]$ from the active source $i$, assuming infinite capacity links, can be characterized by the function $A_{i}\left(t_{1}, t_{2}\right)$. If $A_{i}(t)=A_{i}(0, t)=\sigma_{i}+\rho_{i} t$, then by definition session $i$ starts greedy, i.e., it starts with its maximal burst $\left(\sigma_{i}\right)$ at time zero and continues to transmit with its maximal rate $\left(\rho_{i}\right)$. If all sessions start greedy one gets a greedy GPS system. The main results of [5,6] is revisited here supporting our work:

Suppose that $C_{j}>r$ for every session $j$, where $C_{j}$ is the internal link capacity between the session $j$ leaky bucket and the session $j$ queue, and $r$ is the GPS server capacity. Then, for every session $i$, the maximum delay $D_{i}^{*}$ and the maximum backlog $Q_{i}^{*}$ are achieved (not necessarily at the same time) when every session is greedy starting at time zero, the beginning of a system busy period 2 . Further assuming that for each session $i g_{i} \geq \rho_{i}$, then

$$
Q_{i}^{*} \leq \sigma_{i} \quad \text { and } \quad D_{i}^{*} \leq \frac{\sigma_{i}}{g_{i}} .
$$

The significance of the former result is that for worst-case behavior one 'only' has to analyze a greedy system, which makes the analysis more tractable compared to any arbitrary arrival patterns imposed to the system. Therefore, hereafter in the paper without losing generality we only consider greedy GPS systems, where all sessions start greedy at time zero, the beginning of a system busy period. Since the arrival function in the case of infinite capacity links upper bounds the finite capacity link case, throughout our analytical approach we consider only the former scenario. The transformation of the results obtained under the infinite capacity links to the finite one can be done similarly as shown in 5 .

A large subclass of GPS-based schedulers is the rate-proportional servers (RPS), in which weights are set according to session bandwidth demands. It introduces coupling between bandwidth and delay, i.e., the worst-case delay bound is inversely proportional to the long term allocated bandwidth $(\rho)$. The drawback with this weighting is that if one wants to decrease the delay bound of a

\footnotetext{
${ }^{1}$ a session is said to be backlogged if there are some data in its queue

${ }^{2}$ an interval the server is continuously working
} 
session then the corresponding bandwidth should be increased in order to maintain rate-proportional bandwidth allocation. Clearly, this results in inflexible resource allocation and may lead to waste of network resources.

As an example, assume that one would like to half the worst-case delay for session $i$. Since the worst-case delay is $\sigma_{i} / g_{i}$ where $g_{i}=\phi_{i} / \sum_{j} \phi_{j}, \phi_{i}$ should be doubled, which also results in double guaranteed rate of service for that session. In this way, due to the rate-proportional weighting of sessions, the long term sustainable throughput $\rho_{i}$ should also be multiplied by two. This concludes that the more delay sensitive a session is the more bandwidth it requires. Nevertheless, there can be sessions like voice over IP (VoIP), which are delay sensitive but do not require high bandwidth compared to other sessions. In this case, bandwidth (server capacity) might be wasted for providing acceptable upper bound for delay.

The problem is two-fold. On one hand, the delay bounds for sessions are quite loose, although they are also very simple (see (21). On the other hand, these bounds are inversely proportional to the minimum guaranteed service rate and also to the long term sustainable rate due to the rate-proportional weighting of sessions.

In our approach we propose solutions for both of the above problems. As opposed to the rate-proportional weighting we consider a case when the weight $\phi_{i}$ is set independently of the parameter $\rho_{i}$. This is referred to as non rateproportiona 3 weighting of sessions. After characterizing the behaviour of such a system we provide an algorithmic approach to compute tighter upper bounds for delay than those were derived in [56]. This algorithm universally applies to any arbitrary weight assignment, but due to the fact that only the rateproportional weighting was studied in [5], the tightness of our delay bound cannot be compared in case of arbitrary weighting.

The rest of the paper is organized as follows. In the next section we introduce a novel approach to characterize the service rates of an arbitrary weighted GPS server. Next we present an algorithm to calculate useful (tighter) bounds for worst-case delay based on the dynamics of non rate-proportional GPS server. Finally, a numerical example illustrate our theoretical work and the final section draws conclusions.

\section{Service Rates of a GPS Server}

Now, let us turn on a more formal description of the system behaviour. For simplicity let $r=1$, in other words we consider all the quantities normalized to the server rate $r$. Let $\mathcal{L}=\{L(i) \mid i=1, \ldots, N\}$ be an ordered set of indices in which $L(i)$ means that the session $L(i)$ backlog is cleared as $i^{\text {th }}$ in order. Further, let us denote the time instant by $t_{i}$ when the backlog of session $L(i)$ becomes zero. By definition, let $t_{0}=0$ the start of the system busy period with all greedy sessions. During the interval $\left[0, t_{1}\right)$ every session is served by its

${ }^{3}$ non rate-proportional and arbitrary is used interchangeably through this article, though arbitrary weighting contains rate-proportional weighting as a special case 
minimum guaranteed service rate, i.e., $r_{1}^{j}=g_{j}$. Between $t_{1}$ and $t_{2}$ session $L(1)$ has no backlog, so it is served by $\rho_{L(1)}$. The service rates of the still backlogged sessions during that period are

$$
r_{2}^{j}=g_{j}+\frac{\phi_{j}}{\sum_{i=1}^{N} \phi_{i}-\phi_{L(1)}}\left(g_{L(1)}-\rho_{L(1)}\right), \quad j \in\{1, \ldots, N\} \backslash\{L(1)\} .
$$

The second part on the right hand side comes from the fact that after clearing the backlog of session $L(1)$ the remaining bandwidth $\left(g_{L(1)}-\rho_{L(1)}\right)$ is distributed among the other sessions in proportion to their weights.

Theorem 1. During the time interval $\left[t_{k-1}, t_{k}\right), k=1, \ldots, N$ within the system busy period the backlogged session $j$ is served at a rate

$$
r_{k}^{j}=\frac{\left(1-\sum_{l=1}^{k-1} \rho_{L(l)}\right) \phi_{j}}{\sum_{i=1}^{N} \phi_{i}-\sum_{l=1}^{k-1} \phi_{L(l)}}, j \in\{1, \ldots, N\} \backslash\left\{\bigcup_{m=1}^{k-1} L(m)\right\} .
$$

Proof. The theorem is proved by induction. First, we check that the statement is valid for $r_{m}^{j}$ when $m=2$. From equation (3)

$$
r_{2}^{j}=\frac{\phi_{j}}{\sum_{i=1}^{N} \phi_{i}}+\frac{\phi_{j}}{\sum_{i=1}^{N} \phi_{i}-\phi_{L(1)}} \frac{\phi_{L(1)}}{\sum_{i=1}^{N} \phi_{i}}-\frac{\phi_{j}}{\sum_{i=1}^{N} \phi_{i}-\phi_{L(1)}} \rho_{L(1)} .
$$

Since

$$
\frac{\phi_{j}}{\sum_{i=1}^{N} \phi_{i}}+\frac{\phi_{j}}{\sum_{i=1}^{N} \phi_{i}-\phi_{L(1)}} \frac{\phi_{L(1)}}{\sum_{i=1}^{N} \phi_{i}}=\frac{\phi_{j}}{\sum_{i=1}^{N} \phi_{i}-\phi_{L(1)}}
$$

the following equality holds

$$
r_{2}^{j}=\frac{\left(1-\rho_{L(1)}\right) \phi_{j}}{\sum_{i=1}^{N} \phi_{i}-\phi_{L(1)}}
$$

Now, let us assume that the statement in the theorem is valid for $m=k-1$, which means that

$$
r_{k-1}^{j}=\frac{\left(1-\sum_{l=1}^{k-2} \rho_{L(l)}\right) \phi_{j}}{\sum_{i=1}^{N} \phi_{i}-\sum_{l=1}^{k-2} \phi_{L(l)}}, \quad j \in\{1, \ldots, N\} \backslash\left\{\bigcup_{v=1}^{k-2} L(v)\right\} .
$$

The question is how this rate changes when the session $L(k-1)$ backlog is cleared at time instant $t_{k-1}$. The session $L(k-1)$ service rates are $r_{k-1}^{L(k-1)}$ and $\rho_{L(k-1)}$ respectively before emptying its backlog and after emptying its backlog. In this way the remaining capacity $r_{k-1}^{L(k-1)}-\rho_{L(k-1)}$ is distributed among sessions still backlogged in the system in proportion to their weights. So one can write

$$
\begin{gathered}
r_{k}^{j}=r_{k-1}^{j}+\frac{\phi_{j}}{\sum_{i=1}^{N} \phi_{i}-\sum_{l=1}^{k-1} \phi_{L(l)}}\left(r_{k-1}^{L(k-1)}-\rho_{L(k-1)}\right), \\
\text { where } j \in\{1, \ldots, N\} \backslash\left\{\bigcup_{v=1}^{k-1} L(v)\right\} .
\end{gathered}
$$


Substituting $r_{k-1}^{j}$ from equation (8) it is obtained that

$$
\begin{aligned}
r_{k}^{j} & =\frac{\left(1-\sum_{l=1}^{k-2} \rho_{L(l)}\right) \phi_{j}}{\sum_{i=1}^{N} \phi_{i}-\sum_{l=1}^{k-2} \phi_{L(l)}}+ \\
& +\frac{\phi_{j}}{\sum_{i=1}^{N} \phi_{i}-\sum_{l=1}^{k-1} \phi_{L(l)}}\left(\frac{\left(1-\sum_{l=1}^{k-2} \rho_{L(l)}\right) \phi_{L(k-1)}}{\sum_{i=1}^{N} \phi_{i}-\sum_{l=1}^{k-2} \phi_{L(l)}}-\rho_{L(k-1)}\right) .
\end{aligned}
$$

After some manipulations one gets

$$
r_{k}^{j}=\frac{\left(1-\sum_{l=1}^{k-2} \rho_{L(l)}\right) \phi_{j}}{\sum_{i=1}^{N} \phi_{i}-\sum_{l=1}^{k-1} \phi_{L(l)}}-\rho_{L(k-1)} \frac{\phi_{j}}{\sum_{i=1}^{N} \phi_{i}-\sum_{l=1}^{k-1} \phi_{L(l)}},
$$

which yields the statement of Theorem 1 after simplification.

Corollary 1. $r_{k}^{j}$ is an increasing function of $k$ for every backlogged session.

Proof. Using the formula of (9) we only have to prove that the increment in the iterative formula is greater than or equal to zero. In this case the first factor of the multiplications is strictly greater than zero. Since $L(k-1)$ identifies the session that last emptied its backlog, its backlog function can be expressed in a form

$$
Q_{L(k-1)}(t)=Q_{L(k-1)}(\tau)+\rho_{L(k-1)}(t-\tau)-r_{k-1}^{L(k-1)}(t-\tau)
$$

where: $Q_{L(k-1)}(\vartheta)$ is the session $L(k-1)$ backlog (in units of traffic) at time $\vartheta$ and $\tau$ is the time when session $L(k-2)$ emptied its backlog.

Since at time $\tau$ session $L(k-2)$ emptied its backlog and session $L(k-1)$ is still backlogged, so $Q_{L(k-1)}(\tau)>0$. On the other hand, the next session to empty its backlog is $L(k-1)$, so for some $t>\tau, Q_{L(k-1)}(t)$ will be zero, thus yielding for the inequality $r_{k-1}^{L(k-1)}>\rho_{L(k-1)}$, which proves our corollary.

In order to introduce a theorem defining the maximum backlog of sessions we first declare two lemmas each stating an important attribute of a GPS system.

Lemma 1. For a system busy period in a stable system $\left(\sum_{i} \rho_{i}<r\right)$ where all sessions start greedy, at time zero (beginning of a system busy period) there is at least one session that starts its service with higher rate than its arrival rate.

Proof. This Lemma is proved by contradiction. For a stable system $\sum_{i=1}^{N} \rho_{i}<r$ [5] and the initial service rate, i.e., the guaranteed rate for each session is defined as $g_{i}=\frac{\phi_{i}}{\sum_{i=1}^{N} \phi_{i}} r$. Now assume there exists no session whose guaranteed service rate is higher than its arrival rate, i.e., $g_{i} \leq \rho_{i}, \forall i \in\{1, \ldots, N\}$.

$$
r>\sum_{i=1}^{N} \rho_{i} \geq \sum_{i=1}^{N} g_{i}=r
$$

which is a contradiction, thus ensuring the existence of at least one session with strictly higher service rate than its arrival rate that proves our Lemma. 
Lemma 2. In a system busy period where all sessions start greedy, at any time $t$ there is at least one backlogged session that is served with higher rate than its arrival rate.

Proof. This Lemma is proved by contradiction. At any time $t$ during the system busy period sessions can be divided into two disjoint sets; one corresponding to those already emptied their backlogs $\mathcal{F}(t)$ and the other still having backlogs at time $t$ further denoted by $\mathcal{B}(t)$. At any time $t \mathcal{F}(t) \cap \mathcal{B}(t)=\emptyset$ and $\mathcal{F}(t) \cup \mathcal{B}(t)=$ $\{1, \ldots, N\}$. We still have the stable system criterion, i.e., $\sum_{i=1}^{N} \rho_{i}<r$, however this can be reorganized according to the two sets: $\sum_{i \in \mathcal{F}(t)} \rho_{i}+\sum_{i \in \mathcal{B}(t)} \rho_{i}<r$.

Further denote session $i$ service rate at time $t$ by $r_{i}(t)$ in a way that for $\forall k \in\{1, \ldots, N\}$

$$
r_{i}(t)=r_{k}^{i}, \quad \text { if } t \in\left[t_{k-1}, t_{k}\right) .
$$

Now assume that there exists a $t$ that $r_{i}(t) \leq \rho_{i} \forall i \in \mathcal{B}(t)$, while $r_{i}(t)=$ $\rho_{i} \forall i \in \mathcal{F}(t)$.

$$
r-\sum_{i \in \mathcal{F}(t)} \rho_{i}>\sum_{i \in \mathcal{B}(t)} \rho_{i} \geq \sum_{i \in \mathcal{B}(t)} r_{i}(t)
$$

From Theorem 1 one can write

$$
r_{i}(t)=\frac{\phi_{i}}{\sum_{j \in\{\mathcal{B}(t) \cup \mathcal{F}(t)\}} \phi_{j}-\sum_{j \in \mathcal{F}(t)} \phi_{j}}\left(r-\sum_{j \in \mathcal{F}(t)} \rho_{j}\right)
$$

that is summed for all $i \in \mathcal{B}(t)$ will yield for

$$
\begin{aligned}
\sum_{i \in \mathcal{B}(t)} r_{i}(t) & =\frac{\sum_{i \in \mathcal{B}(t)} \phi_{i}}{\sum_{i \in \mathcal{B}(t)} \phi_{i}+\sum_{j \in \mathcal{F}(t)} \phi_{j}-\sum_{j \in \mathcal{F}(t)} \phi_{j}}\left(r-\sum_{j \in \mathcal{F}(t)} \rho_{j}\right) \\
& =\left(r-\sum_{j \in \mathcal{F}(t)} \rho_{j}\right) .
\end{aligned}
$$

From (17) the contradiction of (15) immediately follows.

Theorem 2. Each session $i$ experiences its maximal backlog either at time zero or at a time when its rate increases (changing).

Proof. From Corollary 1 it follows that there are at most two phases for each session: the first corresponds to the accumulation phase, where the backlog of session $j$ increases and the second that corresponds to backlog emptying phase.

Those sessions starting their services with higher service rates than their arrival rates will only take part in the backlog emptying phase. Since after their initial burst is cleared their backlogs will continuously decrease and their maximal backlog is limited to their initial burst size.

The other set of sessions, which start with smaller service rates than their arrival rates, will step by step increase their rates by each time a session empties its backlog (result of Corollary 1). 
From Lemma 2 it further follows that there's always at least one session clearing its backlog, thus the set of backlogged sessions will decrease ensuring the transition of accumulating sessions to backlog clearing ones. These sessions will experience their maximum backlog at these transition times.

\section{Computing Backlog Clearing Times}

In the previous section we have seen that the time instances when the backlogs are cleared and their orders play a key role in the dynamics of the traffic service. Here we provide an algorithmic approach how to compute the backlog clearing times $t_{k}, k=1, \ldots, N$ and based on these quantities a tighter upper bound for the maximum delay of every session.

As the main result of [5] the maximum backlog and delay of sessions occur (not necessarily at the same time) when all sessions are greedy, i.e., they start to send data at the same time and with maximum possible rate.

In the first step, we should consider the following $N$ equations, which describe the time-evolution of incoming and outgoing (being served) traffic of sessions.

$$
\sigma_{i}+\rho_{i}\left(t-t_{0}\right)=r_{1}^{i}\left(t-t_{0}\right), \quad i=1, \ldots, N \text {. }
$$

On the left-hand side the incoming traffic of session $i$ is expressed until time $t$ and on the right-hand side the amount of served traffic is represented until time $t$ provided the service rate is $r_{1}^{i}$. Assuming the former service rate and the definition of $t_{0}=0$, the time needed for session $i$ to empty its backlog is expressed

$$
t_{i, 1}=\frac{\sigma_{i}}{r_{1}^{i}-\rho_{i}} .
$$

It is clear that among $t_{i}$ 's there can be negative values in case of those sessions whose backlog is temporarily increasing. Apparently, that session clears its backlog first whose $t_{i}$ takes the smallest positive value. More formally

$$
t_{1}=\min \left\{t_{i, 1} \mid t_{i, 1}>0 ; \quad i \in\{1, \ldots, N\}\right\}
$$

and $L(1)$ is the index of session $i$ that satisfies the above minimum.

After session $L(1)$ empties its backlog at time $t_{1}$, there remain $N-1$ backlogged sessions in the server. The service rate of session $i$ backlogged is changed (increased) to $r_{2}^{i}$. In the next (second) step for determining $t_{2}$ consider the following $N-1$ equations:

$$
\sigma_{i}+\rho_{i}\left(t-t_{0}\right)=r_{1}^{i}\left(t_{1}-t_{0}\right)+r_{2}^{i}\left(t-t_{1}\right), \quad i \in\{1, \ldots, N\} \backslash L(1) .
$$

After decomposing $\rho_{i}\left(t-t_{0}\right)$ and solving the equation for $t$ one gets

$$
t_{i, 2}=\frac{\sigma_{i}-r_{1}^{i} t_{1}+r_{2}^{i} t_{1}}{r_{2}^{i}-\rho_{i}}
$$


as the candidate finishing times of still backlogged sessions. Taking the minimum of positive backlog times through $i \in\{1, \ldots, N\} \backslash L(1)$ will yield for the next finishing time of the system

$$
t_{2}=\min \left\{t_{i, 2} \mid t_{i, 2}>0 ; \quad i \in\{1, \ldots, N\} \backslash L(1)\right\}
$$

where $L(2)$ corresponds to the session index $i$ that satisfies the above minimum.

In general, the $k^{\text {th }}$ step can be expressed by the equation

$$
\sigma_{i}+\sum_{j=1}^{k-1} \rho_{i}\left(t_{j}-t_{j-1}\right)+\rho_{i}\left(t-t_{k-1}\right)=\sum_{j=1}^{k-1} r_{j}^{i}\left(t_{j}-t_{j-1}\right)+r_{j}^{i}\left(t-t_{k-1}\right)
$$

Solving the above equation for $t$ yields the $k^{\text {th }}$ step candidate finishing times of still backlogged sessions, i.e.,

$$
t_{i, k}=\frac{\sigma_{i}+\sum_{j=1}^{k-1}\left(\rho_{i}-r_{j}^{i}\right)\left(t_{j}-t_{j-1}\right)+\left(r_{k}^{i}-\rho_{i}\right) t_{k-1}}{r_{k}^{i}-\rho_{i}} .
$$

Since $t_{0}=0$ by definition, $\rho_{i}$ 's can be eliminated from the numerator yielding for the following result

$$
t_{i, k}=\frac{\sigma_{i}+\sum_{j=1}^{k-1} r_{j}^{i}\left(t_{j-1}-t_{j}\right)+r_{k}^{i} t_{k-1}}{r_{k}^{i}-\rho_{i}}
$$

The $k^{\text {th }}$ step finishing time is calculated by taking the minimum of (26) over $i \in\{1, \ldots, N\} \backslash \bigcup_{j=1}^{k-1} L(j)$, i.e.,

$$
t_{k}=\min \left\{t_{i, k} \mid t_{i, k}>0 ; \quad i \in\{1, \ldots, N\} \backslash \bigcup_{j=1}^{k-1} L(j)\right\}
$$

However, to reduce the computation complexity of subsequent $t_{i, k}$ 's, we found the following recursion:

$$
t_{i, k}=\frac{S_{i, k}}{r_{k}^{i}-\rho_{i}},
$$

where $S_{i, k}$ is the numerator of (26) and

$$
S_{i, k+1}=S_{i, k}-r_{k}^{i} t_{k}+r_{k+1}^{i} t_{k}=S_{i, k}+\left(r_{k+1}^{i}-r_{k}^{i}\right) t_{k} .
$$

The calculation of backlog clearing times is quite simple and can be highly automated using our proposed recursive formula. One only has to maintain the rolling sum of $S_{i, k}$ 's, then calculating $r_{k}^{i}$ in each step using (9) and further taking the minimum of (28) to get the next step finishing time. 


\section{Calculating Tighter Upper Bounds for Delay}

The delay bounds of GPS systems - or its packetized versions - have been widely analyzed under leaky-bucket constrained input traffics. However, the simple delay bound (see (2) )

$$
\hat{D}_{i}=\frac{\sigma_{i}}{g_{i}}
$$

where $g_{i}$ is the guaranteed service rate of session $i$ holds only when $g_{i} \geq \rho_{i}$ for session $i$ 6 7]. These sessions are called locally stable sessions, i.e., they do not have backlog accumulating phase [6].

This very constraint however does not allow to set weights $(\phi)$ without restrictions, thus limiting the usable parameter space.

To overcome this limitation, we introduce a theorem to calculate the delay of sessions for any arbitrary weighting. Further, we state that the delay bound we calculate is at least as good as that given by (30), provided locally stable weight assignment.

\section{Theorem 3 (Delay Bounds).}

(i) If $r_{i}(t)$ as defined in (14) satisfies

$$
r_{i}\left(\tau_{i}\right) \geq \rho_{i}
$$

then

$$
D_{i}=\tau_{i}
$$

where $\tau_{i}$ is defined:

$$
W_{i}\left(0, \tau_{i}\right)=\int_{0}^{\tau_{i}} r_{i}(t) \mathrm{d} t=\sigma_{i} .
$$

(ii) If (31) does not hold for session $i$ then it starts with an accumulating phase and denote the order it clears its backlog by $I: i=L(I)$, further there exists a $J \in\{1, \ldots, I-1\}$ such that

$$
\left\{\forall t<t_{J}: r_{i}(t)<\rho_{i}\right\} \wedge\left\{\forall t \geq t_{J}: r_{i}(t) \geq \rho_{i}\right\}
$$

holds, then

$$
D_{i}=t_{J}-\frac{W_{i}\left(0, t_{J}\right)-\sigma_{i}}{\rho_{i}},
$$

where $W_{i}(0, t)$ is the amount of session $i$ traffic served up till time $t$.

Proof. We want to calculate the maximal delay of session $i$, i.e.,

$$
D_{i}=\max _{t}\left\{t-A_{i}^{-1}\left(W_{i}(t)\right)\right\}
$$

where $A_{i}^{-1}(W)$ is the inverse of the arrival function. As we consider worst-case maximal delay, we assume a greedy system, where the arrival function and its inverse are

$$
A_{i}(t)=\sigma_{i}+\rho_{i} t
$$




$$
A_{i}^{-1}=t\left(A_{i}\right)=\max \left\{\frac{A_{i}-\sigma_{i}}{\rho_{i}}, 0\right\} .
$$

Now the delay of session $i$ can be written as

$$
D_{i}(t)=t-\max \left\{\frac{\int_{0}^{t} r_{i}(\tau) \mathrm{d} \tau-\sigma_{i}}{\rho_{i}}, 0\right\}, \forall t \geq 0 .
$$

Since (39) is an increasing function for $t \in\left[0, \tau_{i}\right]$ where $\tau_{i}$ is defined by (33), the smallest $t$ when $D_{i}(t)$ achieves its maximum is $\tau_{i}$, so (39) can be rewritten as

$$
D_{i}(t)=t-\frac{\int_{0}^{t} r_{i}(\tau) \mathrm{d} \tau-\sigma_{i}}{\rho_{i}}, \forall t \geq \tau_{i} .
$$

The delay function $\left(D_{i}(t)\right)$ is continuous and linear between breaking points determined by the backlog clearing times. Hence, it's maximum is achieved at a breaking point where its left and right hand size derivate change sign. Thus, taking the left and right hand side derivation of (40) with respect to $t$, one gets the maximum where the derivate turns from positive to negative. The left hand side derivate is

$$
D_{i}^{\prime-}(t)=1-\frac{r_{i}^{-}(t)}{\rho_{i}}
$$

and the right hand side one is expressed similarly, thus the condition of sign changing can be rewritten as

$$
r_{i}^{-}\left(T_{i}^{*}\right)<\rho_{i}, \quad r_{i}^{+}\left(T_{i}^{*}\right)>\rho_{i} .
$$

Since $r_{i}(t)$ is an increasing function in $t$ (see Corollary 1 ), there are two cases:

(a) If $r_{i}\left(\tau_{i}\right) \geq \rho_{i}$, then (40) is a decreasing function, so the maximal delay is achieved at time $\tau_{i}$, which proves (i).

(b) If $r_{i}\left(\tau_{i}\right)<\rho_{i}$, then $T_{i}^{*}$ satisfying (42) is greater than $\tau_{i}$. However as $r_{i}(t)$ is a step function (see Theorem 1) we seek for a $t_{J}$ finishing time that satisfies (34). Further this $t_{J}$ will also maximize the delay, since the step function of $r_{i}(t)$ will cross $\rho_{i}$ at $t_{J}$, which proves (ii).

Now we have to prove that our delay bound is at least as good as (30).

Theorem 4. We state that

$$
D_{i} \leq \hat{D}_{i},
$$

where $D_{i}$ is defined by Theorem 3 and $\hat{D}_{i}$ is defined by (30).

Proof. Since $\hat{D}_{i}$ holds only if $g_{i} \geq \rho_{i}$, from Theorem 3 we only have to consider case (i). From Corollary 1 it follows that

$$
r_{i}(t) \geq g_{i}, \quad \forall t \in\left[0, \tau_{i}\right],
$$

where $\tau_{i}$ is defined by (33), thus replacing $r_{i}(t)$ by $g_{i}$ in (33) we get

$$
\int_{0}^{\hat{\tau}_{i}} g_{i} \mathrm{~d} t=g_{i} \hat{\tau}_{i}=\sigma_{i}
$$

Now using (44), (45) and Theorem 3, one can write $D_{i}=\tau_{i} \leq \hat{\tau}_{i}=\frac{\sigma_{i}}{g_{i}}=\hat{D}_{i}$, that proves our Theorem. 
Table 1. Parameters and delays of two sessions

\begin{tabular}{|c|c|c|c|c|c|c|c|}
\hline Session parameters & $\sigma \mid \rho$ & $\phi^{1}$ & $\phi^{2}$ & $\phi^{3}$ & $\phi^{4}$ & $\phi^{5}$ & $\phi^{6}$ \\
\hline Session 1 (dashed line) & \begin{tabular}{|l|l|}
1 & .2 \\
\end{tabular} & 2 & 6 & 1 & 12 & 1 & 100 \\
\hline Session 2 (cont. line) & \begin{tabular}{|l|l|}
3 & .6 \\
\end{tabular} & 6 & 2 & 12 & 1 & 100 & 1 \\
\hline \multicolumn{8}{|l|}{ Delays } \\
\hline $\begin{array}{l}D_{1} \\
D_{2}\end{array}$ & & $\begin{array}{l}4 \\
4\end{array}$ & \begin{tabular}{|c|}
$4 / 3$ \\
5
\end{tabular} & \begin{tabular}{|c|}
10 \\
$13 / 4$
\end{tabular} & $\begin{array}{c}13 / 12 \\
5\end{array}$ & \begin{tabular}{|c|}
10 \\
$303 / 100$
\end{tabular} & $\begin{array}{c}101 / 100 \\
5\end{array}$ \\
\hline
\end{tabular}

\section{Numerical Example}

In this section we show a numerical example on our approach to calculate delay and backlog bounds for a GPS node.

Consider a GPS node serving two greedy sessions. The parameters describing the sessions and the applied weights can be seen in Table 1 Note that the set $\phi^{1}$ corresponds to the traditional case where weights are assigned proportional to the long term sustained rates.

Table 1 also shows the calculated delay values using our formula. This gives the base of our comparison. It strikes out immediately that the achievable delays are limited to certain intervals, i.e., $D_{1} \in(1,10]$ and $D_{2} \in(3,5]$. Despite the correlation of the two delays, one can favor any of the sessions to come close to its theoretical minimal delay.

To better understand the the behavior of the GPS node, we present results on the arrival and service functions in Fig. 1.a. Note that same sessions are drawn with same line styles and service functions start from zero while arrival functions start with burste. The figure nicely shows how service rate is increasing at backlog emptying time. The fact that one of the sessions sooner or later empties its backlog and further consumes resources (bandwidth) only as much as its sustained rate $(\rho)$ allows the other session to clear its backlog faster, thus reducing its delay. The different settings of weights determine whose backlog is emptied first.

Since our primary goal was to introduce tighter delay bound, let us investigate the delay behavior of the sessions. Fig. 1. b shows the maximal and the experienced delays in the function of time. Again, the same line style corresponds to one session's maximal delay and delay function in time. It is eye-catching that the delay curves for $\phi^{1}$ coincides. However there may be situations when lower rate sessions have real time delay requirements. Now one has to de-couple bandwidth demands from the actual weights and set $\phi$ 's according to the delay needs. For example, in setting $\phi^{4}$ the small bandwidth session is favored against the high bandwidth one. In this case one may also consider that the increase in worst-case delay for the high bandwidth traffic (from 4 to 5) may worth the significant decrease of low rate traffic's delay (from 4 to $13 / 12$ ). Further, one

${ }^{4}$ the characteristics of session 1 and session 2 are denoted by dashed and continuous lines respectively 

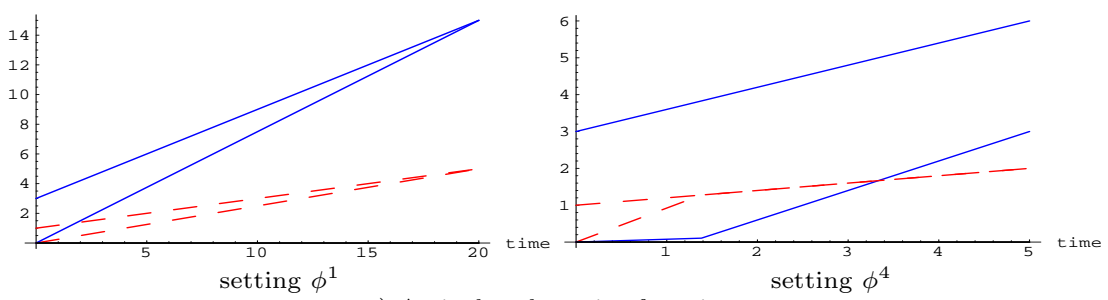

a) Arrival and service functions
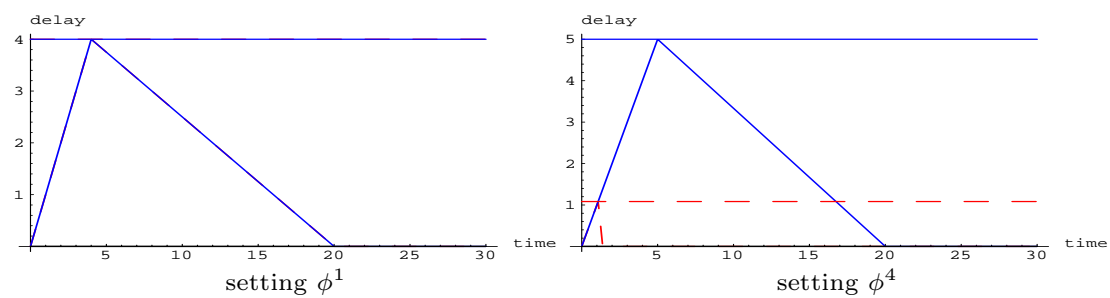

b) Delay functions
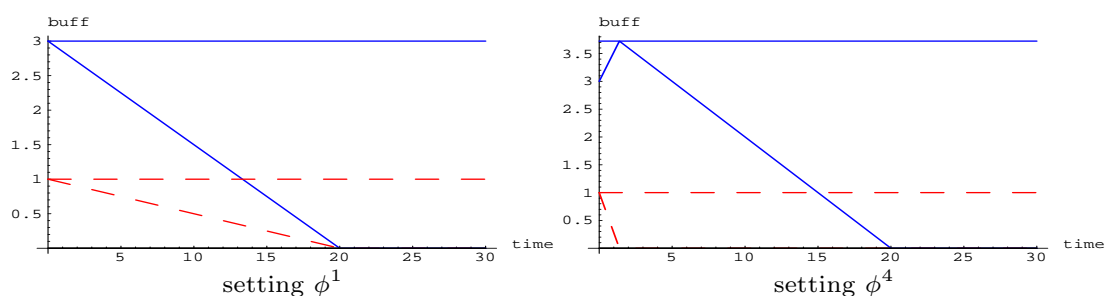

c) Backlog functions

Fig. 1. Session characteristics of an arbitrary weighted GPS node

may carry out a system that is fair in a sense, that smoother traffic gets lower worst-case delay performance than burst ones with indifference of their rates.

It is usually said that there are no gains without losses. The price we have to pay for playing with the weights and setting them arbitrarily is the increase of necessary buffer capacity. Since in the bandwidth delay coupled system the guaranteed service rates are greater than the corresponding arrival rates $\left(g_{i} \geq \rho_{i}\right)$ session backlogs are determined by the burst sizes (see Fig. 1.c setting $\phi^{1}$ ). On the other hand when setting weights de-coupled one of the sessions has to suffer higher delay thus requiring bigger buffers (see Fig. 11.c setting $\phi^{4}$ ).

\section{Concluding Remarks}

In this paper we have analyzed the GPS service discipline with arbitrary weighting of sessions. Although the analysis has been performed for an ideal (fluid) service model the results can be extended to packet-by-packet schedulers in a straightforward manner. We proposed a novel approach to calculate tighter delay bounds under the traditional rate-proportional GPS systems. These bounds are generally tighter than those obtained by Parekh and Gallager in [5], be- 
cause the actual service rate of sessions is taken into account instead of using the guaranteed rate only. The developed algorithm also works in any arbitrary weighted GPS schedulers. A numerical example was presented to detail the behavior of such schedulers. In addition, we have demonstrated that by using arbitrary weighting of sessions the bandwidth delay coupling can be relaxed and worst-case delay bounds can arbitrarily be set between the theoretical bounds.

Besides the tighter delay bound computation under rate-proportional weighting, our work placed emphasis on the assurance of delay bound requirements in such a way that the server capacity is not wasted. This flexibility is achieved by the introduction of non rate-proportional weighting, which also means that the guaranteed fairness may not be valid on all time scale. However, as long as the server is stable, the long term sustainable throughput $(\rho)$ is guaranteed for each session, that is the long term guaranteed fairness is ensured.

The significance of bandwidth delay decoupling and tight performance bounds in existing guaranteed QoS networks is evident. Furthermore, in future Internet services efficient schedulers with predictable performance will also play a key role. Our results can be an important contributions to service models proposed for QoS Internet, too.

\section{Acknowledgement}

This work has been performed in the joint-cooperation between Ericsson - Applied Research Switch Lab and High-Speed Networks Laboratory, Department of Telecommunications and Telematics, Technical University of Budapest. The continuous support of Ericsson is highly appreciated.

\section{References}

1. J.C.R. Bennett and H. Zhang. Hierarchical packet fair queueing algorithms. In Proceedings ACM SIGCOMM'96, pages 143-156, August 1996.

2. D. Clark, S. Shenker, and L. Zhang. Supporting real-time applications in an integrated services packet network: Architetcture and mechanism. In Proceedings of ACM SIGCOMM'92, pages 14-26, Baltimore, Maryland, August 1992.

3. Alan Demers, Srinivasan Keshav, and Scott Shenker. Analysis and simulation of a fair queueing algorithm. In SIGCOMM Symposium on Communications Architectures and Protocols, pages 1-12, Austin, Texas, September 1989. ACM. also in Computer Communications Review, 19 (4), Sept. 1989.

4. L. Kleinrock. Queueing Systems, Volume 2: Computer Applications. Wiley, 1976.

5. A.K. Parekh and R. G. Gallager. A generalized processor sharing approach to flow control in integrated services networks: The single-node case. IEEE/ACM Transactions on Networking, 1(3):344-357, June 1993.

6. A.K. Parekh and R. G. Gallager. A generalized processor sharing approach to flow control in integrated services networks: The multiple node case. IEEE/ACM Transactions on Networking, 2(2):137-150, April 1994.

7. H. Zhang. Service disciplines for guaranteed performance service in packet-switching networks. In Proceedings of the IEEE, 83(10), October 1995. 